\title{
Evaluation de la bioaccumulation du plomb dans les espèces animales marines et identification des sources de contamination métallique par une analyse multiélémentaire en métaux $(\mathrm{Al}, \mathrm{Cd}, \mathrm{Cr}, \mathrm{Cu}, \mathrm{Pb})$ dans les eaux côtières du Bénin
}

\author{
Alassane YOUSSAO ${ }^{1 *}$, Henri H. SOCLO ${ }^{1}$, Clément BONOU ${ }^{2}$ et Benjamin FAYOMI ${ }^{3}$ \\ ${ }^{1}$ Laboratoire d'Etude et de Recherche en Chimie Appliquée, Ecole Polytechnique d'Abomey-Calavi/Université \\ d'Abomey-Calavi, O1BP 2009 Cotonou, République du Bénin. \\ ${ }^{2}$ Laboratoire d'Impact Environnemental, Institut des Sciences Biomédicales Appliquées (ISBA) 01 BP 188 \\ Champ de Foire Cotonou/ Bénin. \\ *Corresponding author; E-mail: abdyoussao@yahoo.fr ; Tel. (00229) 97894320/95396623
}

\section{RESUME}

Différentes études complémentaires ont été menées sur le littoral de l'Océan Atlantique et l'estuaire du Chenal de Cotonou au Bénin, deux zones qui ont des profils et activités différents. La bioconcentration du plomb a été évaluée chez les poissons herbivores (Sphyraena afra, Lutjanus goreensis, Elops lacerta, Lutjanus goreensis et Decapterus rhonchus), les carnivores (Acanthurus monrovia et Pseudupeneus prayensis) et les crevettes (Penaus nottialis). Les concentrations les plus fortes de plomb (ANOVA, p <0.05) ont été obtenues dans les échantillons de crevette de l'espèce penaus nottialis $(0,137 \mathrm{mg} / \mathrm{kg})$ et dans les poissons de l'espèce pseudupeneus prayensis $(0,143 \mathrm{mg} / \mathrm{kg})$ avec des facteurs de bioconcentration (FBc) de 361 et 377 respectivement. Trois sources de métaux ont été identifiées : le Port Autonome de Cotonou (PAC) pour le chrome $(\mathrm{Cr})$ et le cuivre $(\mathrm{Cu})$, l'usine de phosphate d'Hahotoé-Kpogamé pour l'aluminium ( $\mathrm{Al}$ ) et les eaux continentales venant par le chenal de Cotonou pour le plomb $(\mathrm{Pb})$.

(c) 2011 International Formulae Group. All rights reserved.

Mots clés : Bioconcentration, plomb, ANOVA, analyse multi élémentaire, eaux littorales.

\section{INTRODUCTION}

Au Bénin, la pêche est un secteur primordial pour l'économie et pour l'alimentation. Elle contribue pour environ $75 \%$ à la production halieutique nationale et participe pour près de $31 \%$ à la consommation nationale de protéines animales (Gnonhossou, 2006). Ces produits, les plus importants pour la consommation locale, doivent, en outre, satisfaire aux exigences des pays importateurs en matière de sécurité des aliments pour espérer contribuer plus efficacement à la croissance économique. Malheureusement, ces ressources sont affectées par la pollution au Bénin comme dans la plupart des pays africains (Ben Bouih et al., 2004). Le trafic de l'essence frelatée, le trafic routier, les déchets municipaux, industriels, agricoles et artisanaux sont les sources de contamination les plus importantes (Maystre, 1994 ; Soclo et al., 2000; Kouame, 2006). Mais, peu de littératures donnent la preuve scientifique de la contamination de la faune marine (Bouhali et al., 2008), encore moins l'importance du 
trafic de l'essence comme source de plomb. Or, pendant plusieurs décénies, ce trafic détient le monopole de la commercialisation de ce produit et le plomb organique qu'il contient est plus toxique que sa forme inorganique pour les poissons.

Ainsi, les espèces Sphyraena afra, Elops lacerta, Lutjanus goreensis et Decapterus rhonchus chez les poissons carnivores, Acanthurus monrovia et Pseudupeneus prayensis, chez les poissons herbivores et les crevettes de l'espèce Penaus nottialis pêchés dans ces eaux marines ont fait l'objet d'une étude de bioconcentration du plomb; ces espèces sont les plus abondantes de l'ichtyofaune du littoral béninois.

L'objectif de ce travail est d'évaluer le niveau de contamination des compartiments environnementaux (eau, sédiments et ressources halieutiques). Une analyse multiélémentaire en métaux ( $\mathrm{Al}, \mathrm{Cd}, \mathrm{Cr}, \mathrm{Cu}, \mathrm{Pb}$ ) a permis au prime abord d'identifier les sources de contamination métallique dans leur milieu de vie (eau, sédiments) et de calculer le Facteur de Bioconcentration (FBc) relativement au plomb et selon le régime alimentaire.

\section{MATERIEL ET METHODES}

\section{Sites d'échantillonnage et analyses} chimiques

Sept (7) sites ont été échantillonnés, dont quatre (4) sur les côtes de l'Océan Atlantique notés P1, P2 au port autonome de Cotonou (PAC), GP à la plage de Grand Popo, $\mathrm{C}$ à l'embouchure du chenal de Cotonou et trois (3) sur la berge ouest du Chenal de Cotonou notés $\mathrm{C} 1, \quad \mathrm{C} 2$ et $\mathrm{C} 3$ situés respectivement au Lycée technique Coulibaly, à Hindé et à Djidjè.

Le matériel échantillonné est constitué de sédiments, d'eau et d'organismes vivants (poissons et crevettes) sur au total six (6) campagnes d'échantillonnage de septembre 2005 à juin 2006.

Les échantillons d'eau sont prélevés dans des flacons (plastique) tandisque les échantillons de sédiments de surface récupérés à la benne schippeck sont mis dans des emballages plastiques propres et conservés à 4 ${ }^{\circ} \mathrm{C}$ dans des glacières contenant des accumulateurs de froid. Le matériel biologique a été échantillonné dans les prises des pécheurs au Port Autonome de Cotonou (PAC).

Cinq grammes (5 g) d'échantillon de matériel biologique frais sont pesés, broyés et traités avec $10 \mathrm{ml}$ du mélange d'acide nitrique et d'acide perchlorique dans les proportions 6/1 en volume (Andrew et al., 1995) pendant $12 \mathrm{~h}$ pour l'extraction du plomb. L'ensemble du mélange est d'abord porté à $100{ }^{\circ} \mathrm{C}$ pendant 15 à $20 \mathrm{mn}$, puis à $250{ }^{\circ} \mathrm{C}$ jusqu'à disparition des vapeurs nitreuses. Le minéralisât refroidi, est récupéré avec de l'acide nitrique à $10 \%$, puis repris à l'eau distillée à $25 \mathrm{ml}$.

Les échantillons d'eau et de sédiments sont traités conformément à la norme $\mathrm{H} / \mathrm{NF}$ EN ISO 15587-1 et 2 (INERIS, 2003) pour l'extraction des métaux $(\mathrm{Al}, \mathrm{Cd}, \mathrm{Cr}, \mathrm{Cu}, \mathrm{Pb})$ à l'eau régale $\left(\mathrm{HCl}+\mathrm{HNO}_{3}: 3 / 1\right)$.

Ainsi, $20 \mathrm{ml}$ d'eau brute sont prélevées pour $10 \mathrm{ml}$ d'eau régale et l'ensemble a été chauffé à $150{ }^{\circ} \mathrm{C}$ pendant 2 heures pour la minéralisation.

Une masse de $0,5 \mathrm{~g}$ de sédiments a été pesée pour $45 \mathrm{ml}$ d'eau régale et l'ensemble a été porté à $150{ }^{\circ} \mathrm{C}$ pendant 3 à $4 \mathrm{~h}$ afin de bien digérer la matière organique et libérer ainsi les ions $\mathrm{Pb}^{2+}$.

Le minéralisât obtenu dans chaque cas a été filtré et repris à $50 \mathrm{ml}$ à l'eau distillée, puis analysé au spectroscope d'absorption atomique à flamme de type GTA 110 VARIAN pour le dosage du plomb, du cadmium, du chrome, du cuivre et de l'aluminium.

\section{Traitements statistiques et formule du Facteur de Bioconcentration (FBc)}

Le traitement statistique est fait à l'aide du test de Student et de l'analyse de variance avec le logiciel SAS (Statistical Analysis Système). Le test de l'analyse de variance est effectué par proc glm de SAS sur les 
concentrations en plomb des organes de poissons. Les individus sont constitués des différentes espèces d'une part et du régime alimentaitaire d'autre part et les variables concentrations de plomb dans les différents échantillons de poisson analysés a permis de faire des comparaisons dans le test d'analyse de variance.

La formule de calcul du FBc est inspirée de (Casas, 2005) en mode statique. Ce facteur a été déterminé par rapport à l'eau. C'est le rapport entre la concentration interne en plomb dans l'organisme vivant $(\mathrm{Cm})$ et la concentration environnementale (eau) en contaminant $(\mathrm{Cd})$ en $\mathrm{mg} / \mathrm{kg} \mathrm{Pb}$.

$$
F B C=\frac{C m}{C d}
$$

\section{RESULTATS}

Les métaux dans les milieux aquatiques

L'analyse de l'aluminium, du cadmium, du chrome, du cuivre et du plomb a permis de représenter les graphes de la Figure 1(a) relatif aux concentrations des métaux dans les eaux marines et la Figure 1(b) qui ressort la particularité du plomb. Les concentrations en chrome et en cuivre décroissent au fur et à mesure qu'on s'éloigne de P1 situé dans le bassin portuaire, mais le cuivre présente une légère hausse de sa concentration sur le site de Grand Popo (GP). Le test de corrélation entre les sites montre une forte relation $(r=0.96 ; p<0.01)$ entre les concentrations en métaux des sites $\mathrm{P} 1$ et $\mathrm{P} 2$. De plus une corrélation positive et hautement significative existe entre le cuivre et le chrome $(\mathrm{r}=0.9 ; \mathrm{p}<0.01)$.

Sur les sites de l'Océan Atlantique, les concentrations moyennes calculées pour le plomb sont de $1,105 \mathrm{mg} / \mathrm{kg}$ et $1,628 \mathrm{mg} / \mathrm{kg}$ respectivement à $\mathrm{P} 1$ et $\mathrm{P} 2$ dans les sédiments de la zone portuaire, tandis que ces concentrations sont de $0,240 \mu \mathrm{g} / \mathrm{L} \mathrm{Pb}$ et 0,160 $\mu \mathrm{g} / \mathrm{L} \mathrm{Pb}$ dans l'eau. Par contre, le site de l'embouchure (C) dont la concentration est de $0.95 \mu \mathrm{g} / \mathrm{L} \mathrm{Pb}$ est nettement supérieure aux autres valeurs obtenues sur le littoral (ANOVA, p<0.05). D'une manière générale, toutes les valeurs dépassent largement la concentration ubiquitaire qui est de $50 \mathrm{ng} / \mathrm{L}$.

Le plomb présente donc sa plus grande valeur au site $\mathrm{C}$ à l'embouchure du chenal de Cotonou et des concentrations pratiquement uniformes sur tous les autres sites. Les corrélations entre les concentrations de plomb dans l'eau et celle des autres métaux est négative. Une situation similaire se présente avec l'aluminium sur le site de Grand Popo où les coorélations sont toutes négatives et significatives au seuil $p<0.05$. La Figure 1(a) montre en effet que l'aluminium a le taux le plus élevé au site de Grand Popo.

Sur le Chenal de Cotonou, les concentrations obtenues dans l'eau, sur les sites de Hindé $\left(C_{1}\right)$, Djidjè $\left(C_{2}\right)$ et Dantokpa $\left(\mathrm{C}_{3}\right)$ sont respectivement de $0.29 \mathrm{mg} / \mathrm{L}, 0.42$ $\mathrm{mg} / \mathrm{L}$ et $0.65 \mathrm{mg} / \mathrm{L}$, avec une moyenne d'ensemble de $0,45 \mathrm{mg} / \mathrm{L}$ pour l'eau.

Les sites du Chenal de Cotonou montrent donc une nette différence avec les précédents (sites du littoral). La concentration moyenne calculée est de $377,1 \mathrm{mg} / \mathrm{kg}$ de plomb pour les sédiments pour l'ensemble des sorties. Ceux-ci portent alors l'histoire de la pollution en plomb du milieu car concentrent d'importantes quantités du métal.

\section{Le plomb dans les ressources halieutiques}

Les gammes de concentration mesurées vont de $0,025 \mathrm{mg} / \mathrm{kg}$ chez l'espèce lutjanus goreensis (LG) à $0,075 \mathrm{mg} / \mathrm{kg}$ chez l'elops lacerta $(E L)$ dans les poissons carnivores et de $0,011 \mathrm{mg} / \mathrm{kg}$ chez acanthurus monroviae (AM) à $0,143 \mathrm{mg} / \mathrm{kg}$ chez le pseudupeneus prayensis dans les poissons herbivores. Il faut noter une faible dispersion des valeurs autour de la moyenne. L'analyse de variance montre qu'entre les carnivores et les herbivores la différence de concentration n'est pas significative au seuil de 5\%. Il en est de même entre les espèces Sphyraena afra $(S A)$ adultes et jeunes (Figure 4).

Par contre, pseudupeneus prayensis (PP) $(0,140 \mathrm{mg} / \mathrm{kg})$ et les crevettes penaus nottialis $(P N)$ concentrent le plomb de façon significative comparativement aux autres espèces de poisson (ANOVA, p<0.05). 


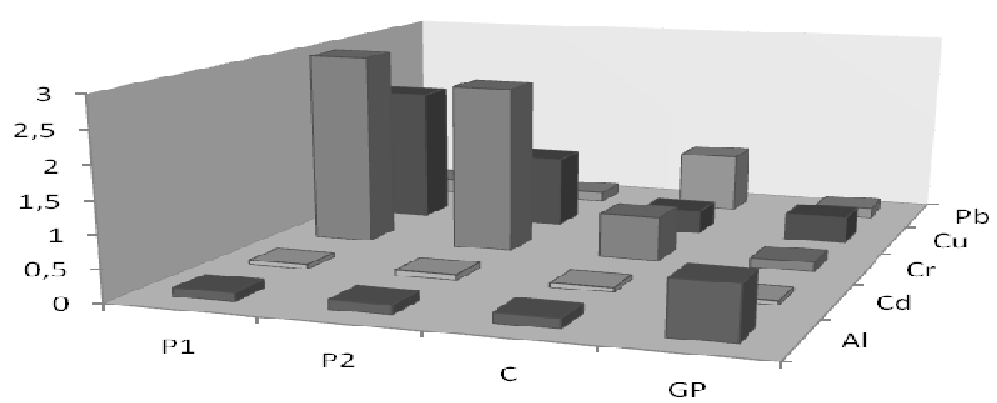

Figure 1a : Distribution des métaux sur les sites du littoral.

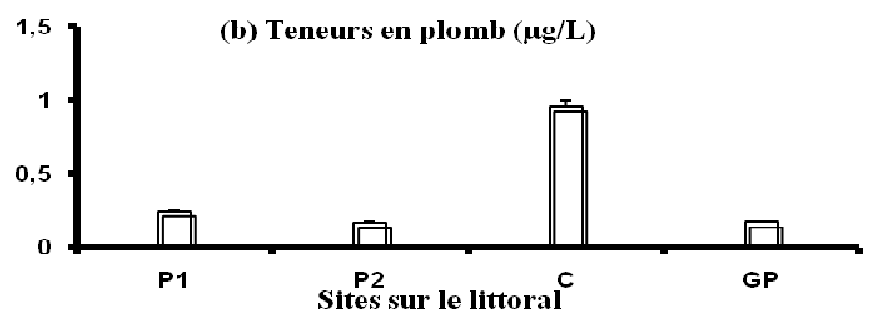

Figure 1b : Distribution du plomb sur les sites du littoral.

(a) Concentrations $(\mathrm{mg} / \mathrm{kg})$

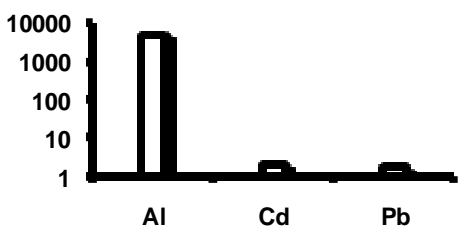

(b) Concentrations $(\mu \mathrm{g} / \mathrm{L})$

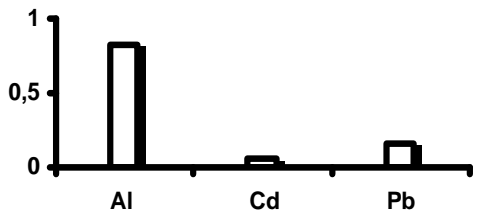

Figure 2 : Concentration métallique (a) dans les boues d'usine de phosphate du Togo et (b) dans l'eau de Grand Popo.

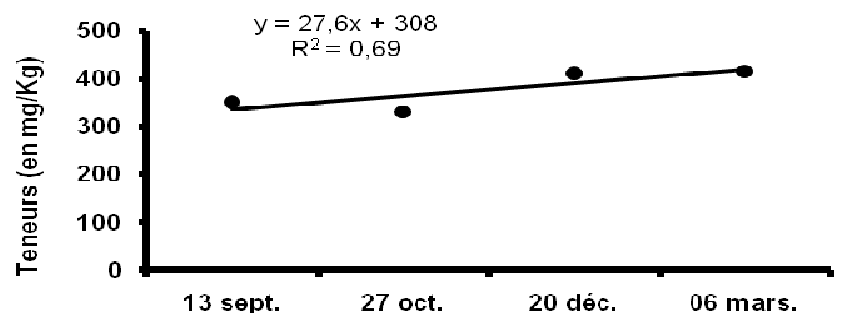

Figure 3 : Evolution des concentrations de $\mathrm{Pb}$ sur le $\mathrm{CC}$. 


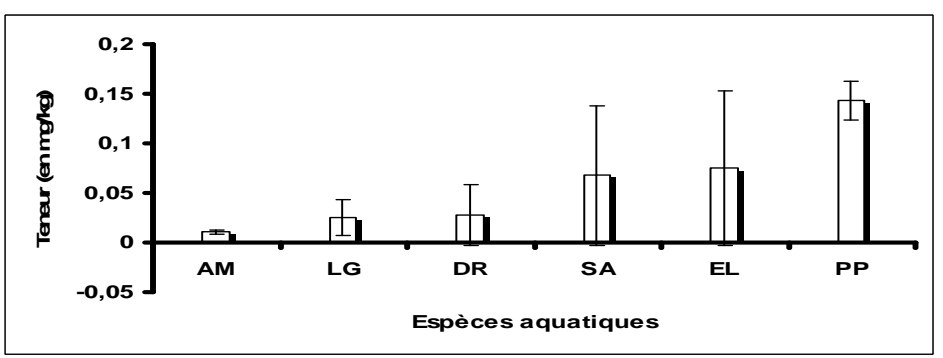

Figure 4 : Distribution du plomb selon l'espèce aquatique (Voir légende). Poissons carnivores : Sphyraena afra (SA) ; Lutjanus goreensis $(L G)$; Elops lacerta (EL); Decapterus rhonchus $(D R)$

Poissons herbivores: Acanthurus monroviae (AM); Pseudupeneus prayensis (PP)

Crevettes : Penaus nottialis $(P N)$

\section{DISCUSSION}

\section{Sources de la contamination métallique}

Cette analyse met en évidence diverses origines de la contamination métallique des eaux côtières du littoral béninois avec trois principales tendances qui se dégagent.

Les teneurs plus élevées de chrome et de cuivre, et la forte corrélation entre P1 et P2 montrent que les activités du Port Autonome de Cotonou sont caractéristiques des apports importants de chrome et de cuivre. Cette source se propage vers l'intérieur de l'Océan (vers P2) et vers les périféries longeant le littoral.

Par contre, la Figure 1(b) met en évidence le Chenal de Cotonou qui s'illustre dans la pollution par le plomb. Ce résultat est conforme à l'analyse du plomb sur les sites $\mathrm{C} 1, \mathrm{C} 2$ et $\mathrm{C} 3$ qui montrent des concentrations 1000 fois plus fortes dans l'eau comparativement aux sites de l'embouchure. De plus, les corrélations négatives confirment cette hypothèse qui signifie que le plomb a une source différente de celle des autres métaux.

Comme indiqué précédemment, la Figure $2(\mathrm{a} \& \mathrm{~b})$ fait apparaitre le transport transfrontalier de pollution métallique. La corrélation négative confirme que la source se situe ailleurs que sur les sites explorés. En effet, les boues de phosphates de HahotoéKpogamé (Togo) dont les concentrations en aluminium $(5409,30 \mathrm{mg} / \mathrm{kg})$, en cadmium $(2,3$ $\mathrm{mg} / \mathrm{kg})$, et en plomb $(1,83 \mathrm{mg} / \mathrm{kg})$ dans les sédiments sont rejetées dans l'océan Atlantique sans aucun tratement préalable selon les travaux de Gnandi (1998) et Bodjock (2003). Les graphes de la Figure 2 (a \& b) qui font un parallèle entre les concentrations de métaux $(\mathrm{Al}, \mathrm{Cd}, \mathrm{Pb})$ dans les boues de traitement de phosphate d'Hahotoé-Kpogamé au Togo et les concentrations métalliques sur le site de Grand Popo pourraient nous éclairer davantage sur les origines de l'aluminium. Mais ce n'est pas suffisant pour une conclusion définitive. Il est donc possible que l'aluminium trouve son origine des activités de l'usine d'Hahotoé-Kpogamé au Togo, mais des analyses isotopiques (Centineo et al., 2004; Komárek et al., 2008) et la présence de boues de phosphates pourraient aider à confirmer cette hypothèse dans des études ultérieures. Toutefois, il n'est pas exclu que d'autres métaux tels que le cadmium et le cuivre soient transportés également de cette source diffuse.

Il faut noter que cette étude préliminaire a déterminé la suite des travaux. Le choix du plomb comme centre d'intérêt pour la recherche et du Chenal de Cotonou comme milieu d'étude se justifie dès lors. En effet, à l'issue de cette analyse multiélémentaire, nous avons engagé un ensemble de travaux documentaire et d'exploration du chenal de Cotonou. L'attention a été focalisée sur les activités sources de contamination en plomb (Abba et al., 2008) et pouvant justifier cet état de 
choses. Entre autres, l'essence chargée en plomb organique (Gallert et al., 2004; Bounit et al., 2005 ; Zúniga et al., 2009), le trafic routier sur les trois ponts érigés sur le Chenal, seuls passages d'est en ouest de la ville et vis versa, les eaux usées urbaines ont été aussi incriminées. Ainsi, la suite des travaux est consacrée à la pollution par le plomb dans le Chenal de Cotonou et l'étude de la contamination des espèces marines les plus rencontrés dans les prises de pécheurs en mer. Toutefois, d'autres études sont envisagées afin d'explorer les autres sites incriminés et les métaux correspondants.

A l'issue de l'analyse précédente, les deux zones d'étude que sont le littoral et l'estuaire du Chenal de Cotonou sont très différentes au vu des gammes de concentration en plomb et des sources de pollution métallique.

Les teneurs élevées de plomb sur le Chenal de Cotonou sont en accord avec la conclusion de l'analyse multiélémentaire indiquant que le chenal est source de contamination des eaux littorales béninoises. Ce phénomène est mis en évidence par la Figure 4. Les valeurs relativement fortes obtenues s'expliqueraient en partie par les critères de choix des sites d'échantillonnage sur le Chenal de Cotonou (CC). En effet, les sites ont été choisis en fonction des activités sources de plomb (Bounit et al., 2005 ; Kouame et al., 2006). Le trafic de l'essence connaît un accroissement important d'année en année et ces produits contenaient une forte quantité de plomb organique (Bouhali et al., 2008) et à ce jour, ce métal n'est pas complètement éliminé de l'essence. De plus, le trafic routier, la source la plus importante selon la littérature (Viard et al., 2004), est de plus en plus intense sur les ponts érigés sur le chenal de Cotonou.

\section{Impact sur la qualité de la vie aquatique}

Il n'y a pas de différence significative entre les facteurs de bioconcentration (FBc) en plomb calculés par rapport à l'eau qui est de 132 chez les poissons carnivores et des herbivores (211). Les valeurs des concentrations dans les échantillons de poissson sont largement inférieures à celles trouvées par Bouhali et al. (2008) chez G. holbrooki qui a obtenu des valeurs qui oscillent entre 400 et 880 ppm.

L'accroissement de la teneur de plomb dans les sédiments au cours de la période d'étude pourrait être élucidé par l'analyse isotopique. On ne saurait conclure sur l'origine de ce plomb, mais le trafic de l'essence doit en être, pour une grande part, la cause. Cependant, l'un des principaux facteurs conditionnant la toxicité aquatique du plomb est la concentration d'ions libres, qui influe sur la biodisponibilité du plomb pour les organismes (Sánchez-Marína et al., 2010 ). Le plomb organique déversé par plusieurs décennies de trafic de l'essence est plus toxique que les composés minéraux de plomb. Une teneur de $1,4 \mathrm{mg} / \mathrm{L}$ (TEL et TML) est mortelle pour les poissons (Convention de Roterdam, 2005). Une étude plus approfondie est nécessaire pour se prononcer sur la raréfaction des ressources halieutiques qui pourrait être liée à cette activité de débarquement de l'essence qui empoisonne assurément la vie aquatique sur le Chenal de Cotonou et dont les effets se font sentir de plus en plus vers l'océan.

\section{Impact sur les ressources halieutiques.}

Les résultats d'analyse montrent que la valeur limite européenne de $0.2 \mathrm{mg} / \mathrm{kg}$ de poids frais dans la chaire de poisson (Règlement CE/78/2005) est loin d'être atteinte. Les valeurs élevées du FBc (132 pour les carnivores et 211 pour les herbivores) sont dues certainement aux faibles valeurs des concentrations dans l'eau.

\section{Conclusion}

La présente étude a permis d'évaluer l'impact de l'activité anthropique sur la qualité des ressources halieutiques et de situer les différentes sources de contamination métallique des eaux du littoral béninois.

Même si le niveau de plomb dans les 
espèces analysées est faible, la présence de ces métaux dans les écosystèmes est une menace sérieuse à prendre en considération dans les études car les conséquences peuvent être d'ordre socio-économique, mais aussi sanitaire. Mais, le cas du chenal de Cotonou reste préoccupant pour le plomb.

\section{REMERCIEMENTS}

Ces travaux de recherche ont été effectués avec le soutien financier de l'Agence Béninoise pour l'Environnement du Bénin (ABE) et de l'International Fundation for Science (IFS).

\section{REFERENCES}

Abba E, Nassali H, Benabid M, El Ayadi R et El Ibaoui H. 2008. Contribution a l'étude physicochimique de l'écosystème lacustre Dayet Aoua au Maroc. Afrique Science, 4(2): 306-317.

Andrew D, Lenor SC, Arnold EG. 1995. Standard Method for the Examination of Water and Wastewater (19 ${ }^{\text {th }}$ edn). Jointly prepared by American Water Works Association (AWWA), Water Environment Federation (WEF) and American Public Health Association (APHA).

Ben Bouih H, Nassali H, Leblans M, Srhiri A. 2004. Contamination en métaux traces des sédiments du lac Fouarat. Afrique Science, 1(1): 109 - 125.

Bodjock K. 2003. Etude chimique de l'impact sur l'environnement du rejet dans la mer des effluents du traitement des phosphates de Hahotoé-Kpogamé (Togo). Mémoire d'Ingénieur de Travaux En Gestion de l'Eau et de l'Environnement (ESTBA)

Bouhali EB, Bennasser L, Nasri I, Gloaguen V, Mouradi A. 2008. Contamination métallique de Gambusia holbrooki au niveau du lac Fouarat et de l'estuaire Sebou dans la région du Gharb (Maroc). Revue Afrique Science, 4(3) : 410 - 425.

Bounit S, ElMeray M. Chehbouni A. 2005. Influence du traitement thermique sur quatre éléments métalliques $(\mathrm{Cu}, \mathrm{Cd}, \mathrm{Pb}$ et $\mathrm{Zn})$ des boues résiduaires. Afrique Science, 1(1) : $69-80$.

Casas S. 2005. Modélisation de la bioaccumulation de métaux traces $(\mathrm{Hg}$, $\mathrm{Cd}, \mathrm{Pb}, \mathrm{Cu}$ et $\mathrm{Zn}$ ) chez la moule, mytilacés galloprovincialis, en milieu méditerranéen. Thèse de l'Université du Sud Toulon VAR.

Centineo G, González EB, Sanz-Medel A. 2004. Multielemental speciation analysis of organometallic compounds of mercury, lead and tin in natural water samples by headspace-solid phase microextraction followed by gas chromatography-mass spectrometry. Journal of Chromatography A, 1034 : 191-197.

Convention de Roterdam. 2005. Application de la procédure de consentement préalable en connaissance de cause aux produits chimiques interdits ou strictement réglementés d'orientation des décisions sur Plomb tétraéthyle et plomb tétraméthyle.

http://europa.eu.int/eur-lex/pri/fr/oj/dat/2001/ I_077/I_07720010316fr00140021.pdf

Gallert C, Winter J. 2004. Degradation of alkyllead compounds to inorganic lead in contaminated soil. Water Research, 38: 4204-4212.

Gnandi K. 1998. Cadmium et autres polluants inorganiques dans les sols et sédiments de la région côtière du Togo : une étude géochimique. Thèse de Doctorat Unique, Université d'Erlangen - Nuremberg, Germany.

Gnonhossou MP. 2006. La faune benthique d'une lagune Ouest Africaine (le lac Nokoué au Bénin), diversité, abondance, variations temporelles et spatiales, place dans la chaîne trophique. Thèse de doctorat de l'Université d'Abomey.169 p.

INERIS. 2003. Institut National de l'Environnement Industriel et des Risques 1 - Plomb et ses dérivés. Version $\mathrm{N}^{\circ} 2$ 1/2003 ERIS-DRC-01-25590-ETSCAPi/SD - N00df257. Fiche de données toxicologiques et environnementales des 
substances chimiques 1/90.

Komárek M, Ettler V, Chrastný V, Mihaljevič M. 2008. Lead isotopes in environmental sciences: A review. Environment International, 3(4): 562-577.

Kouame IK, Gone D, Savane IL, Kouassi EA, Koffi BTA, Goula K, Diallo M. 2006. Mobilité relative des métaux lourds issus de la décharge d'Akouédo et risque de contamination de la nappe du Continental Terminal (Abidjan - Côte d'Ivoire). Afrique Science, 2(1): 39 - 56.

Sánchez-Marína P, Santos-Echeandía J, Nieto-Cid M, Álvarez-Salgado XA, Beiras R. 2010. Effect of dissolved organic matter (DOM) of contrasting origins on $\mathrm{Cu}$ and $\mathrm{Pb}$ speciation and toxicity to Paracentrotus lividus larvae. Aquatic Toxicology, 96: 90-102.

Soclo HH, Guarigues P, Ewald M. 2000. Origin of Polycyclic Aromatic Hydrocarbons (PAHs) in Coastal Marine
Sédiments: Case Studies in Cotonou (Benin) and Aquitaine (France) Areas. Marine Pollution Bulletin, 40(5): 387396.

Viard B, Pihan F, Promeyrat S, Pihan J-C. 2004. Integrated assessment of heavy metal $(\mathrm{Pb}, \mathrm{Zn}, \mathrm{Cd})$ highway pollution: bioaccumulation in soil, Graminaceae and land snails. Chemosphere, 5(5): 13491359.

Zúniga MC, Jovera E, Arancibiab V, Bayona JM. 2009. Development of a methodology for the simultaneous determination of inorganic and organolead compounds using supercritical fluid extraction followed by gas chromatography-mass spectrometry and its application to environmental matrices. Talanta, 80: 504-510. 